\title{
QUANTIFICATION OF ANGIOGENESIS IN THE CHICKEN CHORIOALLANTOIC MEMBRANE (CAM)
}

\author{
SILVIA BLACHER ${ }^{1}$, LAETITIA DEVY ${ }^{1}$, RUSLAN HLUSHCHUK ${ }^{2}$, ETIENNE LARGER $^{3}$, NOEL \\ LAMANDÉ $^{3}$, PETER BURri $^{2}$, PIERRE CORVOL $^{3}$, VALENTIN DJONOV $^{2}$, JEAN-MiCHEL FOIDART $^{1}$ \\ AND AGNES NOËL ${ }^{1}$ \\ ${ }^{1}$ Laboratory of Tumor and Development Biology, University of Liège, Tour de Pathologie (B23), Sart-Tilman; \\ B-4000 Liège; ${ }^{2}$ Institute of Anatomy, University of Berne, Buehlstrasse 26, 3012 Berne, Switzerland; \\ ${ }^{3}$ INSERM U-36-College de France, 11, Place Marcelin Berthelot, 75231 Paris Cedex 05, France \\ e-mail: silvia.blacher@ulg.ac.be \\ (Accepted June 29, 2005)
}

\begin{abstract}
The chick chorioallantoic membrane (CAM) provides a suitable in vivo model to study angiogenesis and evaluate several pro- and anti-angiogenic factors and compounds. In the present work, new developments in image analysis are used to quantify CAM angiogenic response from optical microscopic observations, covering all vascular components, from the large supplying and feeding vessels down to the capillary plexus. To validate our methodology angiogenesis is quantified during two phases of CAM development (day 7 and 13) and after treatment with an antiangiogenic modulator of the angiogenesis. Our morphometric analysis emphasizes that an accurate quantification of the CAM vasculature needs to be performed at various scales.
\end{abstract}

Keywords: angiogenesis, CAM model, image analysis, mathematical morphology.

\section{INTRODUCTION}

The chick chorioallantoic membrane (CAM) is formed by the fusion of the chorion with the allantoic membrane. In this double mesodermic layer, an extremely rich vascular network develops and serves as the respiratory organ of the embryo until the time of hatching. CAM vascularization is characterized by a dense network of arteries and veins from which a delicate and intricate capillary plexus is formed. Initially, the CAM was used as an in vivo assay to study angiogenesis, the budding and sprouting of blood vessels from pre-existing ones. The later process has been shown to take place in numerous physiological and pathological processes (Pepper et al., 1996; Auerbach et al., 1997; Ausprunk and Folkman, 1997; Carmeliet and Collen, 2000; Auerbach et al., 2003; Carmeliet, 2003). More recently, several reports indicate that the process of intussuception, initially described during the postnatal lung development (Caduff et al., 1986; Burri and Tarek, 1990), plays a crucial role in capillary network formation of the CAM (Patan et al., 1993; 1996; Djonov et al., 2000a, b; 2002). Intussuception occurs by internal division of the pre-existing capillary plexus without sprouting. It involves enlargement of capillaries and small supplying vessels which sub-sequently become divided by transluminal tissue pillars. Intussusception leads to vascular beds expansion, increasing capillary network complexity, vascular surface and volume.

The CAM assay provides a suitable model to assess the effects of angiogenic or anti-angiogenic agents. However, the quantification of those effects is not easy. Actually, large vessel-counting methods based on visual examination, manual vessel counts or global measurements of the spatial pattern and distribution of vascular network, is usually used. The following methods have been used to quantify image obtained from light microscope observations: (a) blood vessels enclosed in a silicone ring laid randomly in a treated zone are manually counted (DeFouw et al., 1989; Célérier et al., 2002); (b) binary images of vessels are manually drawn or automatically thresholded and then global parameters like vessel areal density (Rieder et al., 1995), vessel length density (Strick et al., 1991), fractal dimension (Kirshner et al., 1996; Parsons-Wingerter et al., 1998; Vico et al., 1988; Parsons-Wingerter et al., 2000) or complexity measurements (Sandau and Kurz, 1997; Kurz et al., 1998) are determined; (c) individual parameters like the end-points (Strick et al., 1991), number of drawn vessels (Strick et al., 1991) and the length density of radially arranged capillaries during vasculogenesis 
(Jakob and Voss, 1984; Voss et al., 1984) are determined. This last method, which is completely automatic, is appropriated to characterize large changes in vascularity, but unable to quantify more subtle changes. From scanning electronic microscopy, mercox cast images are used to determine automatically vessel areal density and pillar mean area. However, the number and the mean diameter of vessels were still manually determined (Dimitropoulou et al., 1998).

In an attempt to make progress in the complex problem of the quantification of angiogenesis in the CAM model, we present here new developments in automatic image processing and measurements. Optical microscope images at two scales are considered in order to obtain a complete description of the CAM vasculature. To validate our methodology, angiogenesis was quantified during two phases of normal CAM development (days 7 and 13) and after treatment with an antiangiogenic modulator of the angiogenesis, Angiotensinogen (AGT) (Célérier et al., 2002).

\section{MATERIAL AND METHODS}

\section{CHORIOALLANTOIC MEMBRANE ANGIOGENIC ASSAY}

Fertilized White Leghorn chicken eggs were incubated at $37^{\circ} \mathrm{C}$ in a humidified atmosphere $(>60 \%$ relative humidity). By day 3, post-incubation eggs were cracked open and the embryos were deposited in plastic Petri dishes (Auerbach et al., 1974). After 7 days of incubation, a silica ring (10-mm ID) was laid randomly on the CAM for the deposition of testing solution. Each treated animal received the solvent of the tested substance (control treatment) in a second ring laid on the CAM. All protein samples were extemporaneously diluted. Human recombinant angiotensinogen (hAGT) was purified from $\mathrm{CHO}$-cell supernatants as previously described (Célérier et al., 2002) and dissolved in $20 \mathrm{mmol} / \mathrm{L}$ of Tris- $\mathrm{HCl} \mathrm{pH} 8$, $150 \mathrm{mmol} / \mathrm{L} \mathrm{NaCl}$. AGT was applied inside the ring twice at an interval of $24 \mathrm{hrs}$, as previously described (Célérier et al., 2002). Each experimental group contained at least 5 animals. Photographs were taken $48 \mathrm{hrs}$ after the first treatment.

\section{MICROSCOPY AND IMAGE ACQUISITION}

Observations were performed after i.v. injections of $0.1 \mathrm{ml} \mathrm{2.5 \%}$ high molecular weight FITC-dextran ( 70 or $2,000 \mathrm{kDa}$ ) (Sigma) in $0,9 \% \mathrm{NaCl}$ employing a MZ FLIII Leica stereomicroscope, Coolsnap digital camera (Roper) and IPLab software (Scanalytics,
Fairfax, VA). The vehicle-treated area (control) was always compared to the compound-treated area of the same embryo. At least five images of different areas of each animal were analyzed.

\section{IMAGE ANALYSIS}

Image analysis was performed on a $\mathrm{PC}$ using the software 'Aphelion' from Adcis (France). Algorithms developed in this work have been carrying out using traditional tools of signal processing and mathematical morphology (Coster and Chermant, 1985; Soille, 1999). Five images of different areas of the same animal were analyzed. Statistical analysis was performed with MATLAB v.6.5 software.

Depending on the scale of observation, different features can be expected. Figs. 1a,b and 2a,b present CAM images taken at day $13^{\text {th }}$ of development at two different magnifications. At the lowest optical magnifications $(\times 24)$ branching vessels of various sizes and lengths are observed (Figs. 1a,b). At this scale, global measurements like the vessel area and length densities allow characterising the degree of development of the vascular network. For this kind of images, the centripetal ordering method (Fenton et al., 1979) is usually used for classifying vessels (see inset in Fig. 1a). In this method, the smallest vessels that are in contact with the capillary plexus as indicated in Fig. $2 b$ are defined as first-order vessels (Order 1). When two first-order vessels converge an Order 2 vessel is formed (see Fig. $2 b$ and inset of Fig. 1a). Likewise, the convergence of two second-order vessels forms an Order 3 vessel. When two unequal order vessels converge, the highest order is retained. Taking into account that vascular growth (or inhibition) is mainly detected by an increase (or decrease) of the number of vessels that are in direct contact with the capillary plexus, the first-order vessels density seems an accurate parameter to quantify those phenomena. Some first-order vessels are shown in Figs. $1 \mathrm{~b}$ and $2 \mathrm{~b}$.

At higher optical magnification $(\times 60)$, the honeycomb-like structure of the capillary plexus is clearly visible (indicated by arrows in Fig. 2b). Black holes, corresponding to nonvascularized regions, can be observed more clearly in the vicinity of the first order vessels. It has been shown that the size of the nonvascularized regions can be influenced by the presence of pro- or anti-angiogenic factors (Drexler et al., 2000).

In the next sections the proposed algorithms for preliminary image processing and quantification of images at the two scales described above (low and high optical magnification images), are presented. The whole procedure of image processing and measurements for both vessels and nonvascularized regions is schematised in Fig. 3. 

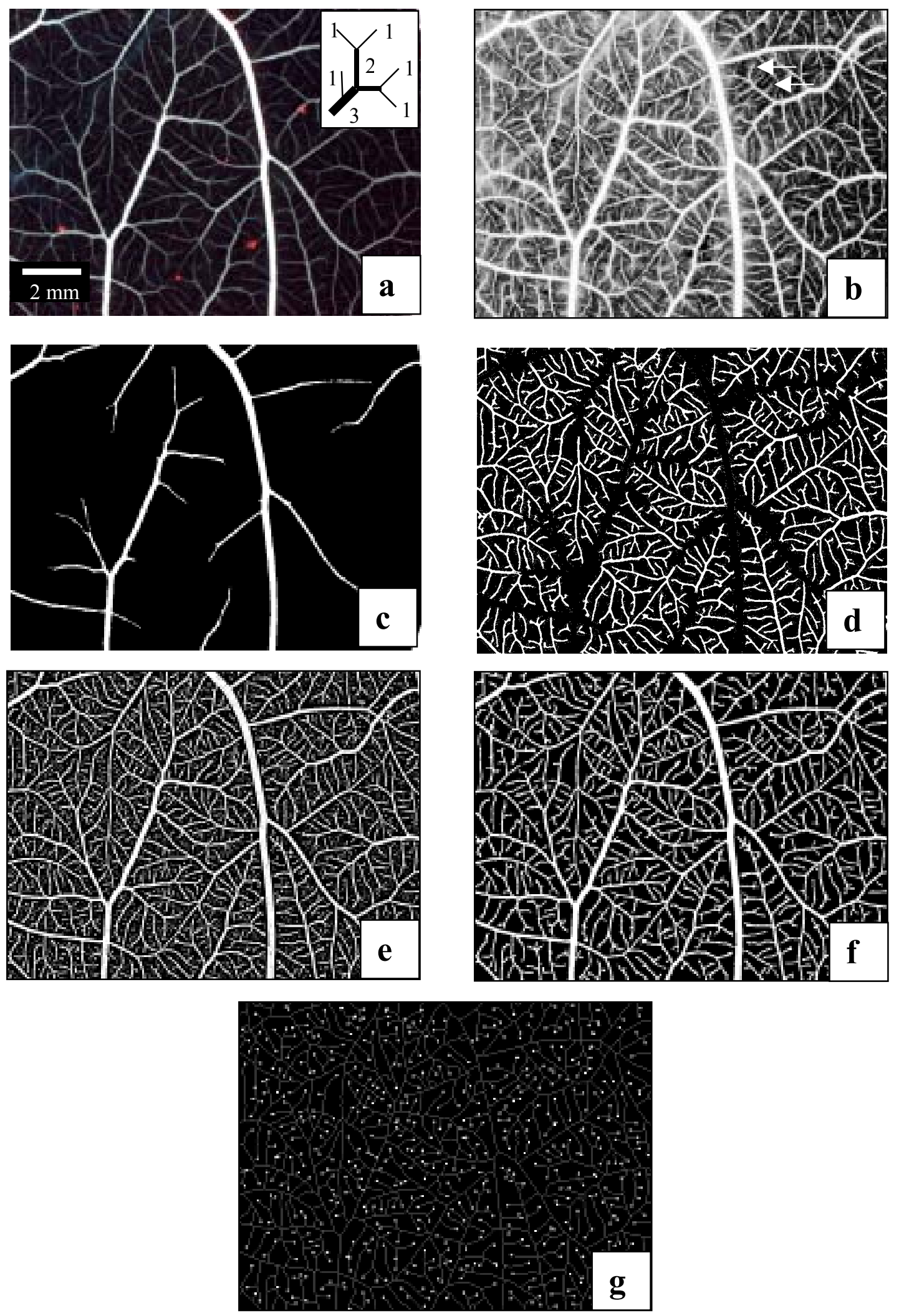

Fig. 1. Binary processing of vessel images at low magnification (x24). (a): Original $R G B$ colour image, and principle of centripetal ordering of the vessels, (b): histogram equalization of the green channel image. Arrows indicate the end of some first-order vessels, (c): extraction of large vessels, (d) extraction of small vessels (venules and arterioles), (e): noisy binary image in which some vessels are disconnected, (f): final binary image, $(g)$ : skeletonized binary image in which dots represent the end-point vessels ( $g$ ). 

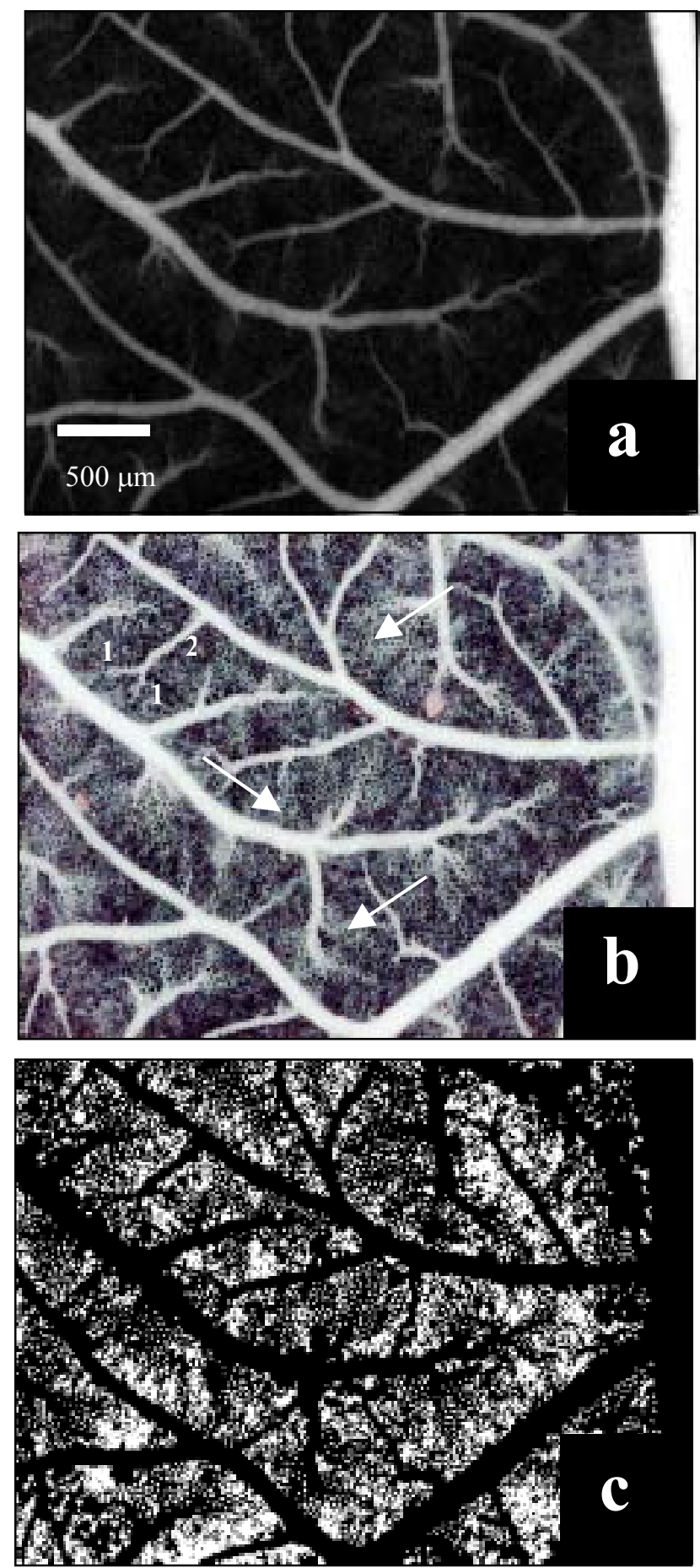

Fig. 2. Binary processing of capillary plexus images: (a) original $R G B$ colour images at $\times 60$ optical magnification, (b): histogram equalization of the green channel image, arrows indicate the capillary plexus, two order 1 and one order 2 vessels are also indicated, (c) binary processed image. 


\section{Image processing and measurements of CAM Images}

\section{Low magnification images}

Images are registered in the full-colour red, green, blue (RGB) space.

(Fig. 1a)

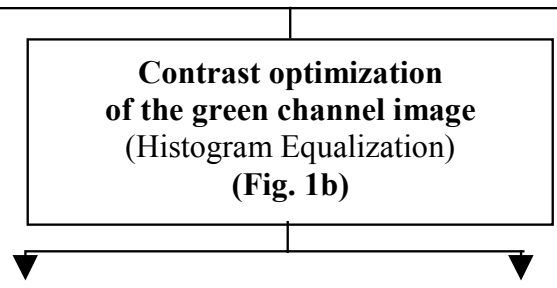

Extraction of large vessels

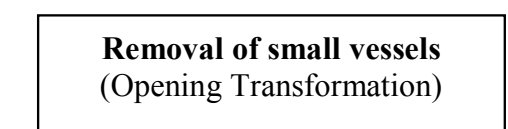

Large vessel binarization (Threshold transformation) (Fig. 1c)

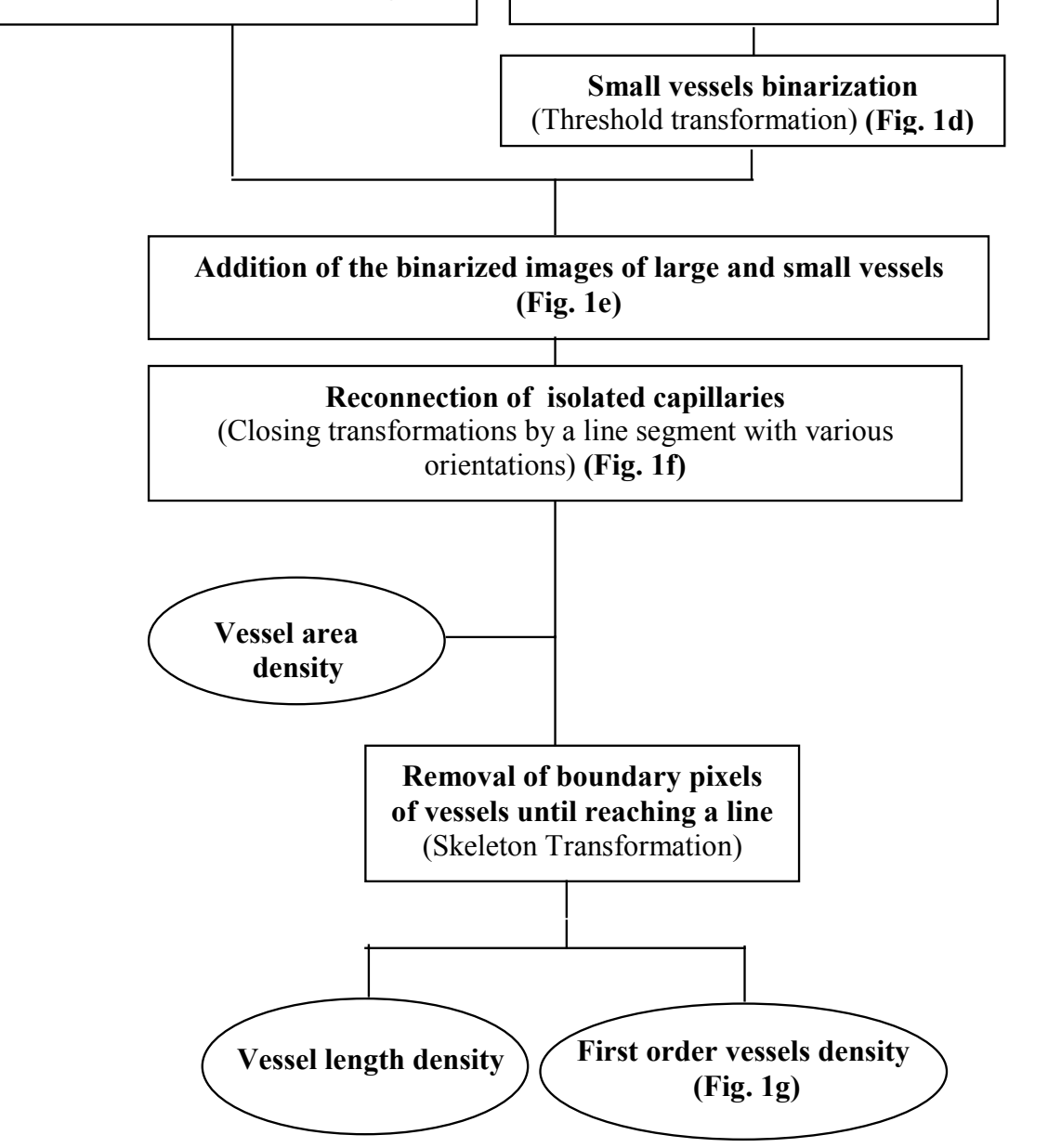

Extraction of small vessels

High magnification images

Images are registered in the full-colour red, green, blue (RGB) space.

(Fig. 2a)

Contrast optimization of the green channel image

(Histogram Equalization)

(Fig. 2b)

Binary extraction of nonvascularized regions (Detection of regional minima) (Fig. 2c)

Removal of large vessels by masking them by their binarized images
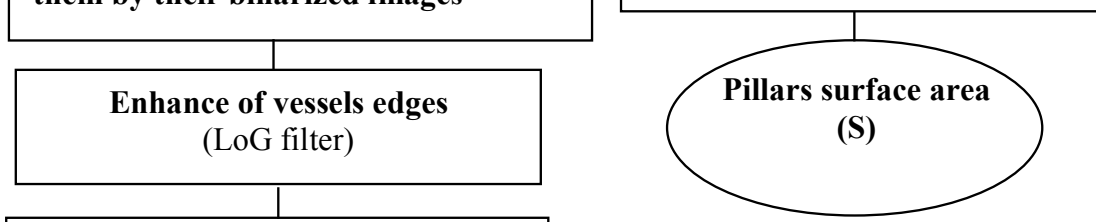

Fig. 3. Schematic representation of image processing and measurements of CAM vascularisation at two magnifications. 


\section{PROCESSING AND MEASUREMENTS OF VESSELS OBSERVED AT LOW OPTICAL MAGNIFICATION}

Images are registered in the full-colour red, green and blue (RGB) space (Fig. 1a). In this space, the vessels are the most contrasted and continuous in the green channel. However, non-linear effects introduced during digitalisation prevent to distinguish small vessels. In order to enhance the image contrast, the histogram equalisation of the grey level intensities is performed (Fig. 1b). At this low magnification $(\times 24)$, a large set of vessels can be observed, and statistically relevant results can be obtained. The first step consists in applying a binary mask on the original grey level image in order to eliminate the capillary plexus background, which appears darker than vessels. The binary mask is obtained by performing a low-level threshold transformation of the original image. Binary image processing is performed by a two-step procedure consisting in the successive extraction of large and small vessels (venules and arterioles) according to the intensity of the fluoresceinated Dextran injected into CAM vessels. To extract large vessels, the small vessels are first filtered out using an opening transformation. Then an automatic threshold technique, which consists in selecting the threshold that maximises the global average contrast of edges (Kohler, 1981) is applied (Fig. 1c).

To extract small vessels, large vessels are masked using as mask the inverse of the previously obtained binary image. Then, to detect small vessel edges, the convolution of the image with a Laplacian of a Gaussian blurring filter (LoG filter) (Marr and Hildreth, 1980), is applied. This process is equivalent to taking the difference between two Gaussian filters images with different standard deviation (DoG filter; Burt and Adelson, 1983). As vessels present a variety of sizes, $5 \times 5 / 3 \times 3$ and $3 \times 3 / 1 \times 1$ kernels were considered. Finally, the image was thresholded assigning a value of 1 to all pixels with positive grey level, and a value of 0 to the others (Fig. 1d). After superposition of large and small vessel binary images (Fig. 1e), some isolated capillaries are reconnected to the vascular network, by computing the maximum of closing transformations by a line segment of 5 pixel $(0.05 \mathrm{~mm})$ long oriented in several directions from $0^{\circ}$ to $180^{\circ}$. Finally every isolated object smaller than an arbitrary size fixed at 5 pixels $(0.05 \mathrm{~mm})$ is removed. Fig. 1f shows the resulting binary image after complete image processing. To characterize the obtained binary image, the following global parameters are determined: (a) the vessels area density defined as the number of pixels that belong to the vascular network on the image divided by the total area of the image, (b) the vessel length density per area defined as the number of pixels that belong to the skeleton of the vascular network on the image divided by the total area of the image and (c) the first-order vessels density per area, defined as the number of vessel extremities divided by the total area of the image.

End-points are determined from the skeleton of the binary image using the appropriate hit-or-miss transform (Coster and Chermant, 1985). Previously, artifacts (i.e., 'prunes') resulting from small distortions on the edges were eliminated from the skeleton. Endpoints corresponding to Fig. 1f are represented in Fig. 1g.

\section{PROCESSING AND MEASUREMENTS OF THE NONVASCULARIZED REGIONS OBSERVED AT HIGHER OPTICAL MAGNIFICATION}

At a higher magnification $(\times 60)$, the structure of the capillary plexus can be observed (Fig. 2b). In this image, nonvascularized regions correspond to regional minima. As the grey levels of "holes" are not homogeneous, minima are taken in a prefixed grey level range. After thresholding, nonvascularized regions appear as white 'grains' (Fig. 2c) and their surface size distribution is determined.

\section{RESULTS}

\section{EVALUATION OF VASCULAR CHANGES DURING NORMAL GROWTH OF THE CAM}

The CAM vasculature at 7 and 13 days of incubation was first quantified from images obtained at $\times 24$ magnification. Representative resulting processed images for those days are illustrated in Fig. 4. At day 7, CAM vasculature is mainly characterized by large vessels with only few branching (Fig. 4a). The histogram equalization shows (Fig. 4b) that first order vessels are thin, tortuous and merge in the capillary plexus. At this point, the identification of the endpoints is complex because the transition from a vessel to the capillary plexus is progressive. Such a problem of end-point determination has been already reported by Strick et al. (1991) who called "blind-ended" the vessels that are connected to the capillary plexus. On the contrary, at day 13, a low magnification image reveals the typical vessel hierarchy of CAM vasculature (Figs. 4d,e). At this stage, end-points are clearly defined and can be more easily determined. Fig. $4 \mathrm{c}$ and $\mathrm{f}$ show 
the resulting binary images from which the end-points density was determined.

Quantification at low magnification, i.e., at the vessel level, reveals that all the measured parameters increase significantly from day 7 to day 13 (see Table 1).

Images taken at $\times 60$ magnification (Figs. 5a and c) show clearly an evolution of capillary mesh during the CAM development. As the measured surfaces of nonvascularized regions spread on a very large interval for both days 7 and 13, distribution are represented in a logarithm scale (Fig. 5e). Statistical parameters for these distributions are presented in Table 2. A Kolmogorov-Smirnov test (KS-test) indicates that the surface distributions of the two populations differ significantly. As the distributions are both grossly non-normally-distributed, percentiles are used to give an idea of the centrality and spread. For the sake of completeness the mean is also included in Table 2.
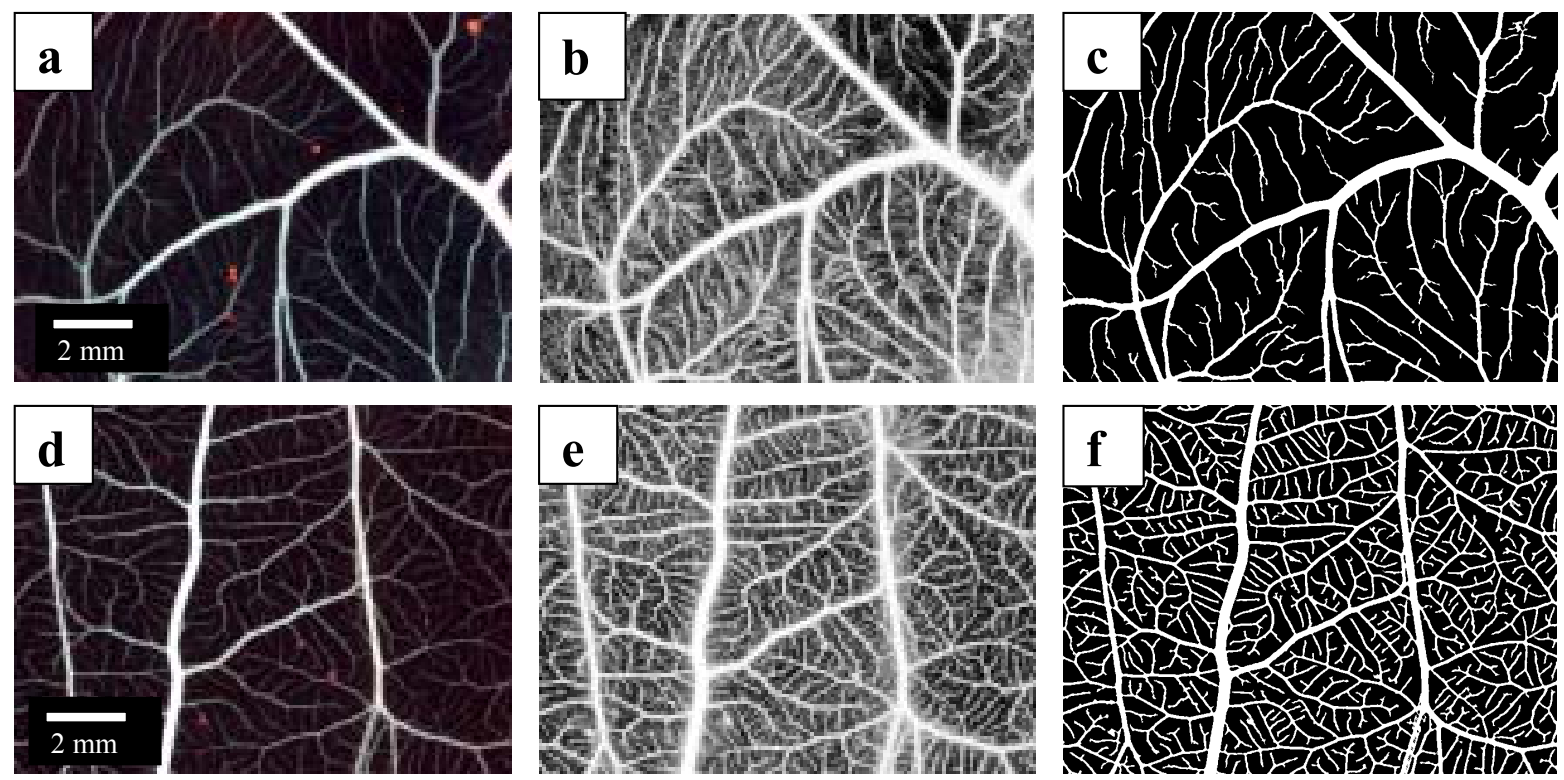

Fig. 4. Original color images taken at day 7 (a) and day 13 (d) of CAM development, at x24 magnification. (b, e): Histogram equalization of green channel images $(a)$ and $(d)$, respectively. $(c, f)$ : Binary processed images obtained from the original images (a) and (d), respectively.

Table 1. Vascular changes observed at low magnification (x24) between days 7 and 13 of the normal development of CAM and between control animals and after AGT inhibitor treatment.

\begin{tabular}{l|c|c|c}
\hline Sample & $\begin{array}{c}\text { Vascular Area } \\
\text { density }\end{array}$ & $\begin{array}{c}\text { Vascular Length } \\
\text { Density } \\
\left(10^{-3} 1 / \mathrm{mm}\right)\end{array}$ & $\begin{array}{c}\text { Vessel End-Points } \\
\text { Density } \\
\left(1 / \mathrm{mm}^{2}\right)\end{array}$ \\
\hline Normal growth (Day 7) & $0.17 \pm 0.03$ & $0.14 \pm 0.02$ & $1.92 \pm 0.16$ \\
Normal growth (Day 13) & $0.32 \pm 0.07$ & $0.30 \pm 0.03$ & $5.07 \pm 0.80$ \\
Control & $0.29 \pm 0.01$ & $0.31 \pm 0.04$ & $5.84 \pm 2.02$ \\
AGT & $0.24 \pm 0.04$ & $0.25 \pm 0.03$ & $3.09 \pm 1.08$ \\
\hline
\end{tabular}

Table 2. Statistical parameters of capillary mesh surface distributions obtained at high magnification (x60) for days 7 and 13 of the normal development of CAM and for control animals and after AGT inhibitor treatment.

\begin{tabular}{l|c|c|c|c|c}
\hline Sample & $\begin{array}{c}\mathrm{x}_{25}{ }^{*} \\
\left(10^{2} \mu \mathrm{m}^{2}\right)\end{array}$ & $\begin{array}{c}\mathrm{x}_{50 *}{ }^{* *} \\
(\text { median) } \\
\left(10^{2} \mu \mathrm{m}^{2}\right)\end{array}$ & $\begin{array}{c}\mathrm{x}_{75} * * * \\
\left(10^{2} \mu \mathrm{m}^{2}\right)\end{array}$ & $\begin{array}{c}\text { Mean } \\
\left(10^{2} \mu \mathrm{m}^{2}\right)\end{array}$ & KS-test \\
\hline Normal growth (Day 7) & 2.786 & 4.830 & 10.077 & 10.457 & $\mathrm{P}<0.05$ \\
Normal growth (Day 13) & 1.300 & 2.229 & 4.644 & 7.452 & 14.336 \\
\hline Control & 5.0155 & 9.102 & 16.718 & $\mathrm{P}<0.05$ \\
\hline AGT & 8.731 & 18.576 & 43.282 & 60.889 & \\
\hline
\end{tabular}

$*: 25^{\text {th }}$ percentile; $* *: 50^{\text {th }}$ percentile; $* * *: 75^{\text {th }}$ percentile; KS-test: Kolmogorov-Smirnov test. 

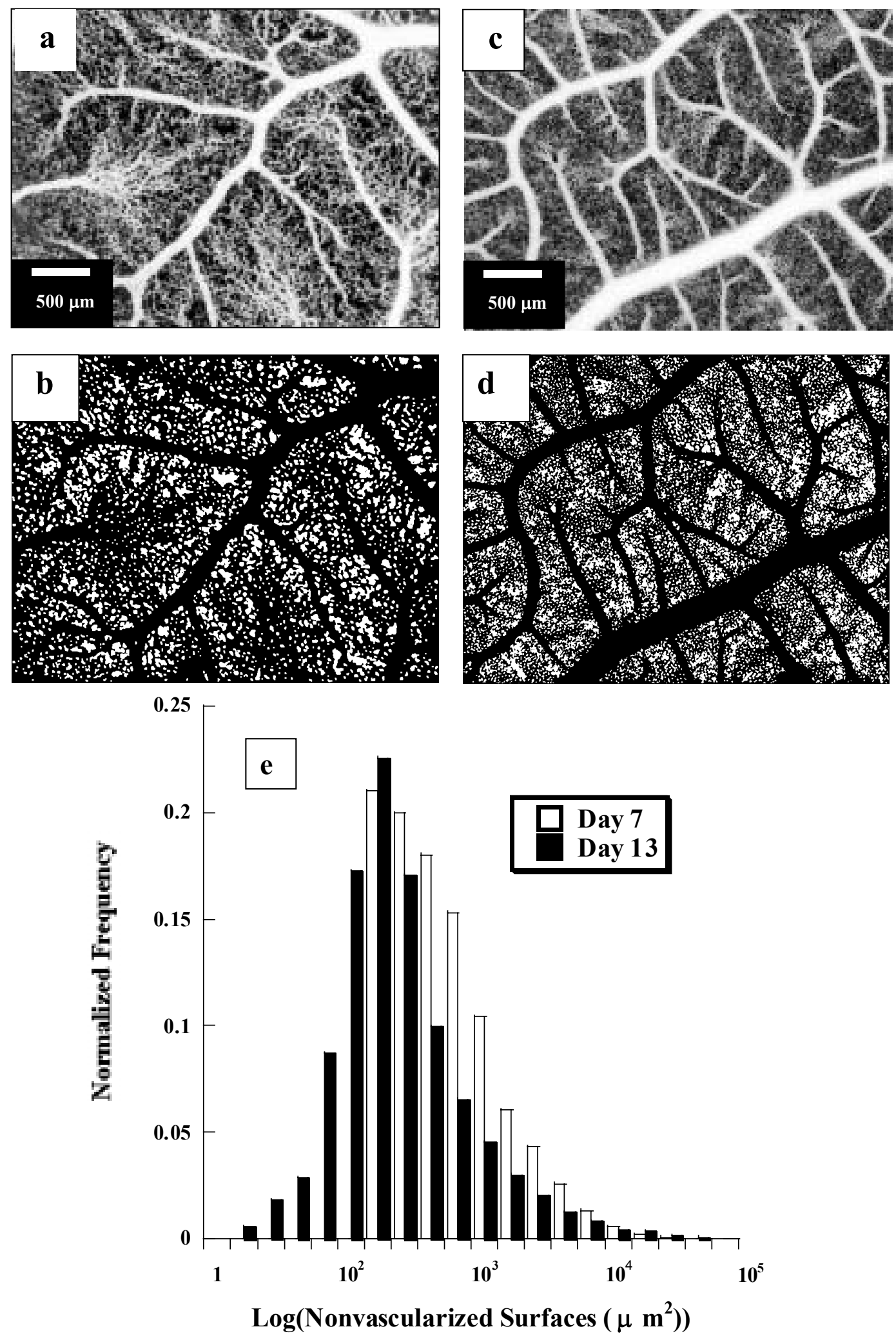

Fig. 5. Capillary plexus images at x60 magnification at day 7 (a), and day 13 (c) of CAM development. Corresponding binary images of the nonvascularized regions for day 7 (b) and day 13 (d). (e) Surface distribution of nonvascularized regions for days 7 and 13. 

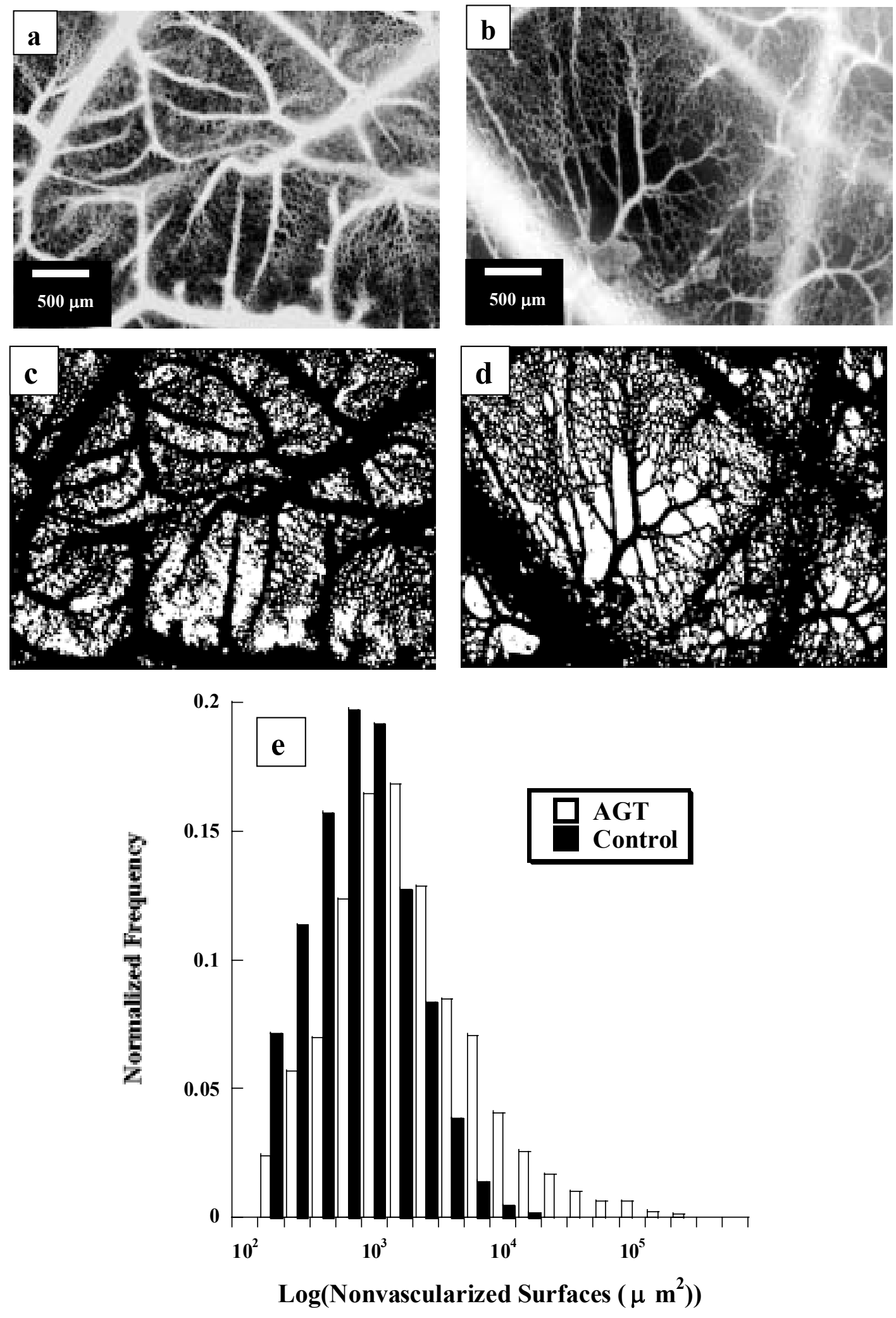

Fig. 6. Capillary plexus images at $\times 60$ magnification for control (a) and AGT-treated (b) animals. Corresponding binary images of the nonvascularized regions for control (c), and AGT treated (d) animals. (e) Surface distribution of nonvascularized regions for control and AGT treated animals. 
A comparison of the three quartiles of the distributions, $\mathrm{x}_{0.25}, \mathrm{x}_{0.50}$ and $\mathrm{x}_{0.75}$, shows that the area of the nonvascularized regions at day 7 is approximately two times larger that at day 13. Moreover, the interquartile range, $\mathrm{x}_{0.75}-\mathrm{x}_{0.25}$, evolves from $7.2910^{2} \mu \mathrm{m}^{2}$ at day 7 to $3.3410^{2} \mu \mathrm{m}^{2}$ at day 13 indicating a decrease of the area dispersion. These observations indicate that at day 7 , the capillary plexus is a heterogeneous honeycomb-like structure composed of meshes with a broad size distribution. From day 7 to 13 , nonvascularized regions shrink in a way that the capillary network organization becomes more homogeneous. This latter arrangement is characteristic of a mature CAM structure.

\section{EVALUATION OF VASCULAR CHANGES UPON AGT INHIBITOR TREATMENT}

Vascular changes were evaluated at low (x24) and high (x60) magnifications on control animals and after modulation by human angiotensinogen AGT. Measurements of the vessel surface area, length and end-points densities show a significantly decrease after treatment with AGT (see Table 1). These results are in agreement with previous manual measurements (Célérier et al., 2002). Representative high magnification images of the control and the treated animals and the surface distributions of the non-vascularized regions are shown in Figs. 6a,b. Statistical parameters are presented in Table 2. A KS-test indicates that the area distributions of the two populations differ significantly. The interquartile range increases from $11.7010^{2} \mu \mathrm{m}^{2}$ for the control to $34.5510^{2} \mu \mathrm{m}^{2}$ for the treated animal, for which the distribution spreads towards the largest values (Table 2 and Fig. 6e). This indicates clearly that AGT treatment gives rise to a more heterogeneous capillary plexus than that observed in the control sample. The presence of the large structures contributes strongly to the mean value that is five times greater than the control counterpart. The measurements performed at low and high magnifications show that the inhibitor affects the structure of the CAM both at vessel and capillary plexus levels.

\section{DISCUSSION}

The CAM assay is classically used in order to study in vivo the vasculature development and the effects of pro- or anti-angiogenic agents. Objective assessment of useful angiogenic parameters is difficult to achieve. Quantifications on this model are usually performed manually at low magnification by counting all or some order vessels. Since such a manual quanti- fication means extremely tedious work, digital image analysis has been used to determine global parameters (surface and length vessel densities or the fractal dimension). In the present work, we propose an objective method of quantification adapted to the various magnifications at which CAM vascular network development can be observed. At low magnifications, global parameters such as vessel surface, vessel length and vessel end-points densities can be determined. Endpoints represent the connection between large vessels (first order vessel visualized at low magnification) and capillary plexus (evidenced at high magnification). Consequently, they can be determined only if the used magnification is low enough not to detect the small capillary extensions that connect the first order vessels to the capillary plexus.

The accuracy in the determination of the vessel surface area, vessel length and end-points densities depends not only on the magnification but also on the stage of CAM development. Indeed, at the early stage of growth (up to day 7), large vessels contribute mainly to the surface and length densities. In this case, the used magnification must be low enough to consider a homogeneous image, i.e., an image containing almost the entirety of the CAM. If not, the results will depend on the particular region of the membrane arbitrarily chosen in which large vessels could be or not present. Similarly, end-points are not easily detected and quantified at the early stage of development. At this stage, the first order vessels are hardly formed, i.e., they are small and thin and merge in the plexus. At a later stage of CAM development (day 13) global parameters mentioned above can be determined more accurately. However, the vessels are so well developed that end-points evaluation is certainly distorted by threedimensional effects.

On the contrary, the image processing of nonvascularized regions observed at high magnification is more straightforward than the processing at vessel level. Indeed, its quantification accounts merely to detect on the images the black spots. In this work, the size distribution of nonvascularized regions was measured. It should be noted that since size distribution of the nonvascularized regions depends on experimental condition (state of CAM development, application of an angiogenic modulator), the used magnification allowing to observe the more potent structural modifications must be chosen according to the specific phenomena studied.

The methodology proposed in this paper was firstly tested at two stages of the normal CAM growth (days 7 and 13). According to previous studies (Strick et al., 1991), these days are an important develop- 
mental window during which the ultimate vascular structure is established. Both low and high optical magnification measurements indicated a transition between immature and mature vasculature. Indeed, low magnification measurements show a growth of vessel area, vessel length and end-points densities between days 7 and 13. This observation is consistent with Strick et al. (1991) results. On the other hand, analysis at high magnification reveals that between days 7 and 13, the capillary plexus evolves from a heterogeneous structure towards homogeneous structure formed by small nonvascularized regions with the narrowest size distribution.

From our studies on the effect of the AGT inhibitor on the CAM development, it appears that the vascular network observed at low and high resolution is significantly modified. Indeed, global parameter values measured on treated animals are smaller than those measured on animals non treated. This result is in agreement with previous works (Célérier et al., 2002). This behavior is accompanied by a modification of the structure of the capillary plexus that becomes more heterogeneous. A comparison with the results obtained from measurements on the normal growth of the CAM, shows that angiogenic inhibitor AGT would have a regressive effect on the natural growth.

\section{ACKNOWLEDGMENTS}

This work was supported by grants from the Communauté française de Belgique (Actions de Recherches Concertées), the Commission of European Communities (FP5 n ${ }^{\circ}$ QLK3-CT02-02136, FP6 $\mathrm{N}^{\circ}$ LSHC-CT-2003-503297), the Fonds National de la Recherche Scientifique (FNRS, Belgium), the Fédération Belge Contre le Cancer, the C.G.R.I.F.N.R.S.-INSERM Coopération, the Fonds spéciaux de la Recherche (University of Liège), the Fortis Banque Assurances, the D.G.T.R.E. from the "Région Wallonne", the Interuniversity Attraction Poles Programme - Belgian Science Policy (Brussels, Belgium). Dr. Yannick Anguy and Mr. Cédric Gommes are kindly acknowledged for their critical reading of the manuscript.

\section{REFERENCES}

Auerbach R, Gilligan B, Lu LS, Wang SJ (1997). Cell interactions in the mouse yolk sac: vasculogenesis and hematopoiesis. J Cell Physiol 173(2):202-5.

Auerbach R, Lewis R, Shinners B, Kubal L, Akhtar N (2003). Angiogenesis assays: a critical overview. Clin Chem 49(1):32-40.

Auerbach R, Kubai L, Knighton D, Folkman J (1974). A simple procedure for the long term cultivation of chicken embryos. J Dev Biol 41(2):391-4.
Ausprunk DH, Folkman J (1997). Migration and proliferation of endothelial cells in preformed and newly formed blood vessels during tumor angiogenesis. Microvasc Res 14(1):53-65.

Burri PH, Tarek MR (1990). A novel mechanism of capillary growth in the rat pulmonary microcirculation. Anat Rec 228(1):35-45.

Burt PJ, Adelson EH (1983). The Laplacian pyramid as a compact code. IEEE Trans Commun COMM 31:337-45.

Caduff JH, Fischer LC, Burri PH (1986). Scanning electron microscope study of the developing microvasculature in the postnatal rat lung. Anat Rec 216(2):154-64.

Carmeliet P (2003). Angiogenesis in health and disease. Nat Med 6:653-60.

Carmeliet P, Collen D (2000). Molecular basis of angiogenesis. Role of VEGF and VE-cadherin. Ann N Y Acad Sci 902:249-62.

Célérier J, Cruz A, Lamandé N, Gasc JM, Corvol P (2002). Angiotensinogen and its cleaved derivatives inhibit angiogenesis. Hypertension 39(2):224-8.

Coster M, Chermant JL (1985). Précis d'analyse d'images. Paris: CNRS.

DeFouw DO, Rizzo VJ, Steinfeld R, Feinberg RN (1989). Mapping of the microcirculation in the chick chorioallantoic membrane during normal angiogenesis. Microvasc Res 38(2): 36-47.

Dimitropoulou C, Malkusch W, Fait E, Maragoudakis ME, Konerding MA (1998). The vascular architecture of the chick chorioallantoic membrane: sequential quantitative evaluation using corrosion casting. Angiogenesis 2: 255-63.

Djonov V, Schmidt M, Tschanz SA, Burri PH (2000a). Intussusceptive angiogenesis: its role in embryonic vascular network formation. Circ Res 86(3):286-92.

Djonov VG, Galli A B, Burri PH (2000b). Intussusceptive arborization contributes to vascular tree formation in the chick chorio-allantoic membrane. Anat Embryol 202(5): 347-57.

Djonov V, Cresto N, Aebersold DM, Burri PH, Altermatt HJ, Hristic M, et al. (2002). Tumor cell specific expression of MMP-2 correlates with tumor vascularisation in breast cancer. Int J Oncol 21(1):25-30.

Drexler HCA, Risau W, Konerding M (2000) Inhibition of proteasome function induces programmed cell death in proliferating endothelial cells. FASEB 66(14):65-77.

Fenton BM, Zweifach BW, Worthen DM (1979) Quantitative morphometry of conjunctival microcirculation in diabetes mellitus. Microvasc Res 18(2):153-66.

Jakob W, Voss K (1984). Utilisation of images analysis for the quantification of vascular reponses in the chick chorioallatoic membrane. Exp Path 26:23-99.

Kohler R (1981). A segmentation system based on thresholding. Computer Graphics and Image Processing. 15:319-38. 
Kurz H, Wilting J, Sandau K, Christ B (1998). Automated evaluation of angiogenic effects mediated by VEGF and PIGF homo- and heteropolimes. Microvasc Res 55:92-102.

Marr D, Hildreth E (1980). Theory of edge detection. Proceeding of the Royal Society of London. B207: 187-217.

Parsons-Wingerter P, Lwai B, Yang MC, Elliot KE, Milaninia A, Redlitz A, et al. (1998). A novel assay of angiogenesis in the quail chorioallantoic membrane: Stimulation of bFGF and inhibition by angiostatin according to fractal dimension and grid intersection. Microvasc Res 55: 201-14.

Parsons-Wingerter P, Elliot KE, Farr AG, Radhakrihnan K, Clark JI, Sage EH (2000). General analysis reveals that TGF $\sim 1$ inhibits the rate of angiogenesis in vivo by selective decrease in the number of vessels. Microvasc Res 59:221-32.

Patan S, Haenni B, Burri PH (1993). Evidence for intussusceptive capillary growth in the chicken chorioallantoic membrane (CAM). Anat Embryol 187(2): 121-30.

Patan S, Haenni B, Burri PH (1996). Implementation of intussusceptive microvascular growth in the chicken chorioallantoic membrane (CAM): 1. pillar formation by folding of the capillary wall. Microvasc Res 51(1): 80-98.

Pepper MS, Montesano R, Mandriota SJ, Orci L, Vassalli JD (1996). Angiogenesis: a paradigm for balanced extracellular proteolysis during cell migration and morphogenesis. Enzyme Protein 49(1-3):138-62.

Rieder MJ, O’Drobinak DM, Greene AS (1995). A computerized method for determination of microvascular density. Microvasc Res 49:180-9.

Sandau K, Kurtz H (1997). Measuring fractal dimension and complexity- an alternative approach with an application. J of Micr 186:164-76.

Soille P (1999). Morphological image analysis. Berlin, Heidelberg, New-York: Springer-Verlag.

Strick DM, Waycaster RL, Montani JP, Gay WJ, Adair TH (1991). Morphometric measurements of chorioallantoic membrane vascularity: effects of hypoxia and hyperoxia. Am J Physiol 260(4):1385-9.

Vico PG, Kyriacos S, Heymans O, Louryans S, Cartilier L (1988). Dynamic Study of the "Extraembrionic vascular network of the chick embryo by fractal analysis". J Theor Biol 195:525-32.

Voss K, Jakob W, Roth K (1984). A new image analysis method for the quantification of neovascularisation. Exp Path 26:155-61. 